\title{
The crosstalk between autophagy and apoptosis: where does this lead?
}

\author{
Claire Gordy, You-Wen He \\ Department of Immunology, Duke University Medical Center, Durham, NC 27710, USA \\ $\triangle$ Correspondence: youwen.he@duke.edu \\ Received November 11, 2011 Accepted December 6, 2011
}

\begin{abstract}
Recent advances in the understanding of the molecular processes contributing to autophagy have provided insight into the relationship between autophagy and apoptosis. In contrast to the concept of "autophagic cell death," accumulating evidence suggests that autophagy serves a largely cytoprotective role in physiologically relevant conditions. The cytoprotective function of autophagy is mediated in many circumstances by negative modulation of apoptosis. Apoptotic signaling, in turn, serves to inhibit autophagy. While the mechanisms mediating the complex counter-regulation of apoptosis and autophagy are not yet fully understood, important points of crosstalk include the interactions between Beclin-1 and $\mathrm{Bcl}-2 / \mathrm{Bcl}-\mathrm{xL}$ and between FADD and Atg5, caspase- and calpain-mediated cleavage of autophagy-related proteins, and autophagic degradation of caspases. Continued investigation of these and other means of crosstalk between apoptosis and autophagy is necessary to elucidate the mechanisms controlling the balance between survival and death both under normal conditions and in diseases including cancer.
\end{abstract}

\section{KEYWORDS autophagy, apoptosis, Beclin-1, Iympho- cytes}

\section{INTRODUCTION}

Macroautophagy (hereafter referred to as autophagy) is a catabolic process whereby cells degrade their own components by enveloping them in double-membrane vesicles referred to as autophagosomes and targeting them for lysosomal degradation. Studies of autophagy in different cell types and under different conditions have provided conflicting results regarding the influence of autophagy on cell death (reviewed by Debnath et al., 2005; Kroemer and Levine, 2008).

Autophagy may be observed in dying cells, and many studies have reported "autophagic cell death" in experimentally manipulated cells or diseased cells undergoing excessive levels of autophagy. For example, inhibition of the proapoptotic protease caspase 8 results in cell death that is associated with enhanced autophagy, and knocking down autophagy-related genes appears to rescue the death of these cells (Yu et al., 2004). Moreover, the increased autophagic vacuole formation that occurs in Parkinson's disease has been attributed to an autophagic cell death process (Anglade et al., 1997).

Despite these reports, autophagy has never been shown to be the cause of death in mammalian cells under physiologically relevant conditions, and many of the published reports of autophagic cell death can be explained by the failure to properly define autophagy and the misinterpretation of the assays commonly used to measure autophagy (Kroemer and Levine, 2008). Instead, accumulating evidence points to a pro-survival function for autophagy through the disposal of damaged organelles, clearance of intracellular infections, or recycling of nutrients to conserve energy under starvation conditions.

Though the term "autophagy" was first used to describe the process by which cells digest organelles and recycle their components in 1963, the molecular mechanisms that underlie autophagy only began to be identified in the late 1990s (Klionsky, 2007; Yang and Klionsky, 2010). The identification of the proteins involved in the initiation and progression of autophagy has begun to reveal the complex interactions between the pathways controlling autophagy and those regulating apoptosis, providing insight into the seemingly opposing reports of autophagic cell death and pro-survival functions for autophagy. 


\section{Box 1. Extrinsic and intrinsic apoptosis}

Apoptosis can be triggered by intrinsic signals such as DNA damage or by extrinsic signals that engage death receptors on the cell surface. Both pathways result in cell death mediated by the activation of cysteine proteases referred to as caspases; however, the upstream events leading to caspase activation are distinct.

Intrinsic apoptosis, which requires the permeabilization of the mitochondrial membrane, is closely regulated by members of the $\mathrm{Bcl}-2$ protein family. The pro-apoptotic Bcl2 family members Bax and Bak serve to permeabilize the mitochondrial membrane, leading to the release of cytochrome c, caspase activation, and cell death. This pro-death function is opposed by the anti-apoptotic Bcl-2 family members $\mathrm{Bcl}-2, \mathrm{Bcl}-\mathrm{x}$, and $\mathrm{Mcl}-1$. A third group of $\mathrm{Bcl}-2$ proteins, referred to as $\mathrm{BH} 3$-only proteins, serves a pro-apoptotic function; however, the precise mechanisms by which these proteins contribute to apoptosis are not clear. These proteins have been proposed to either bind and inhibit the anti-apoptotic Bcl-2 family members or directly interact with and enhance the activity of the proapoptotic Bcl-2 family members.

Extrinsic apoptosis is initiated by the binding of a death receptor, such as Fas, TNFR1, or TRAIL, to its ligand, which results in the formation of the death-inducing signaling complex (DISC). Adapter proteins (FADD and/or TRADD) bind to the cytoplasmic tail of the death receptor through DD-DD interactions and subsequently recruit pro-caspase 8 to the DISC through DED-DED interactions. Recruitment of pro-caspase 8 to the DISC allows for its homodimerization and cleavage, resulting in the release of active caspase 8, which cleaves downstream substrates, including effector caspases, leading to DNA fragmentation and cell death.

Here we will review recent findings demonstrating the crosstalk between apoptotic and autophagic signaling pathways and discuss the implications of these interactions on our understanding of related diseases and therapeutic strategies.

\section{DISCOVERY OF Beclin-1 AS A Bcl-2-INTERACTING PROTEIN AND AN INITIATOR OF AUTOPHAGY}

Until the 1990s, autophagy was understood largely in descriptive terms, as studies focused on morphological analysis of cells undergoing autophagy. While these early studies of mammalian cells delineated the steps of autophagosome formation and lysosome fusion on a structural level, the discovery of autophagy in yeast paved the way for genetic studies that identified the genes involved in what was found to be a highly conserved process. Through genetic screens of autophagy-deficient yeast, the first autophagy gene, Atg1, was identified in 1993 and cloned in 1997 (Tsukada and Ohsumi, 1993; Matsuura et al., 1997).

Shortly thereafter, Beclin-1, the mammalian ortholog of the yeast protein Atg6, was identified in a yeast two-hybrid screen for interacting partners of $\mathrm{Bcl}-2$, the prototypic member of the $\mathrm{Bcl}-2$ family of apoptosis-regulating proteins (Liang et al., 1998). Bcl-2 family proteins share up to four Bcl-2 homology $(\mathrm{BH})$ domains and can be divided into three groups based on the number of these domains and their pro- or anti-apoptotic functions: multi-domain anti-apoptotic $\mathrm{Bcl}-2$ family proteins (Bcl-2, Bcl-xL, Bcl-w, A1, and Mcl-1), multi-domain proapoptotic Bcl-2 family proteins (Bax, Bak, and Bok), and pro-apoptotic $\mathrm{Bcl}-2$ family members that contain only the $\mathrm{BH} 3$ domain, which are thus referred to as BH3-only proteins (Bim, Bid, Bad, Bmf, Puma, Noxa, Bik, Hrk, and Mule). The multidomain pro-apoptotic Bcl-2 family members Bax and Bak serve to permeabilize the mitochondrial outer membrane, allowing for release of cytochrome $c$ and subsequent caspase activation, while the anti-apoptotic $\mathrm{Bcl}-2$ family members function to inhibit these pro-apoptotic proteins. Two models have been proposed for the role of the $\mathrm{BH} 3$-only proteins in this process. In one model, the $\mathrm{BH}$-only proteins are thought to function as sensitizers by binding to anti-apoptotic $\mathrm{Bcl}-2$ family members, thereby reversing their repression of the multi-domain pro-apoptotic Bcl-2 proteins. In the second model, the $\mathrm{BH} 3$-only proteins are suggested to directly interact with the multi-domain pro-apoptotic proteins (Zhang et al., 2005; Youle and Strasser, 2008).

In initial studies, Beclin-1 was shown to play a role in antiviral host defense and to function as a tumor suppressor (Liang et al., 1998; Aita et al., 1999). A subsequent study demonstrated that Beclin-1 was a functional ortholog of the yeast autophagy protein Atg6 that functioned in the induction of autophagy in mammalian cells (Liang et al., 1999). In this study, expression of Beclin-1 was shown to restore autophagy in both Atg6-deficient yeast and MCF7 breast cancer cells (Liang et al., 1999). The reduced tumorigenicity of Beclin-1expressing MCF7 cells in nude mice further suggested that the tumor suppressor function of Beclin-1 was mediated by its ability to induce autophagy (Liang et al., 1999).

Further studies of the molecular mechanism by which Beclin-1 initiates autophagy revealed that Atg6/Beclin-1 interacts with the class III phosphoinositide 3-kinase (PI3K) Vps34 to initiate autophagosome formation both in yeast and in mammalian cells (Kihara et al., 2001a, 2001b). This function appears to be highly conserved, as Beclin-1 has been shown to be required for the initiation of autophagy in not only yeast and mammalian cells, but also plants, Caenorhabditis elegans and Dictyostelium (Meléndez et al., 2003; Martin and Baehrecke, 2004; Otto et al., 2004; Liu et al., 2005). Further evidence for the conserved role of Beclin-1 was provided by data demonstrating that a highly conserved 
region of Beclin-1 is essential for binding to $\mathrm{Vps} 34$ and initiating autophagy (Furuya et al., 2005).

\section{Beclin-1 AT THE NEXUS OF AUTOPHAGY AND APOPTOSIS}

The initial discovery of Beclin-1 as a Bcl-2 binding partner hinted that the Bcl-2-Beclin-1 complex may serve as a point of crosstalk between the apoptotic and autophagic signaling pathways. Indeed, later studies demonstrated that Beclin-1 is a BH3-only protein that also interacts with $\mathrm{Bcl}-\mathrm{xL}, \mathrm{Bcl}-\mathrm{w}$, and $\mathrm{Mcl}-1$ but does not interact with the pro-death $\mathrm{BH} 3$-only members of the Bcl-2 family (Feng et al., 2007; Oberstein et al., 2007). Much like other BH3-only proteins, Beclin-1 interacts with $\mathrm{Bcl}-2, \mathrm{Bcl}-\mathrm{xL}$, and $\mathrm{Mcl}-1$ through its $\mathrm{BH} 3$ domain, and mutations in this domain or in the $\mathrm{BH} 3$ receptor domain in anti-apoptotic $\mathrm{Bcl}-2$ family members prevent this interaction (Feng et al., 2007; Maiuri et al., 2007; Oberstein et al., 2007).

Interestingly, this interaction inhibits Beclin-1-mediated induction of autophagy in nutrient-sufficient conditions in a location-dependent manner. Although Bcl-2, Bcl-xL, and Mcl1 are largely localized to the mitochondria, where they serve to inhibit mitochondrial outer membrane permeabilization, only endoplasmic reticulum (ER)-localized $\mathrm{Bcl}-2$ family members can inhibit starvation-induced autophagy (Pattingre et al., 2005; Maiuri et al., 2007). While the reasons for the subcellular localization-dependent ability of $\mathrm{Bcl}-2$ family members to inhibit the autophagic function of Beclin-1 remain unclear, recent findings suggest the involvement of the novel $\mathrm{Bcl}-2$ binding partner nutrient-deprivation autophagy factor-1 (NAF-1), which appears to stabilize the Bcl-2-Beclin-1 interaction at the ER (Chang et al., 2010). Importantly, knocking down NAF-1 resulted in disruption of the Bcl-2Beclin-1 interaction and induction of autophagy, further demonstrating the inhibitory role of $\mathrm{Bcl}-2$ family members in Beclin-1-mediated autophagy (Chang et al., 2010).

During starvation or other stress conditions, however, Bcl-2 and Bcl-xL must be displaced from Beclin-1 to permit autophagy (Pattingre et al., 2005; Wei et al., 2008). The dissociation of this complex can be achieved through JNKmediated phosphorylation of $\mathrm{Bcl}-2$, death-associated protein kinase (DAPK)-mediated phosphorylation of Beclin-1, translocation of the nuclear protein high-mobility group box 1 (HMGB1) to the cytosol, or competition with other BH3-only proteins for Bcl-2 binding (Luo and Rubinsztein, 2007; Maiuri et al., 2007; Wei et al., 2008; Zalckvar et al., 2009a, 2009b; Kang et al., 2010; Tang et al., 2010).

Nutrient deprivation results in the activation of the stressinduced MAPK JNK, which phosphorylates three residues in the regulatory loop of $\mathrm{Bcl}-2$, disrupting its interaction with Beclin-1 (Wei et al., 2008). This process is essential for the induction of autophagy in nutrient-poor conditions, as cells with either mutations in these phosphorylation sites or deficiency in JNK fail to undergo starvation-induced autophagy (Wei et al., 2008). Moreover, expression of constitutively active JNK is sufficient to induce autophagy in nutrientsufficient conditions in cells expressing wild type $\mathrm{Bcl}-2$ but not in cells expressing a non-phosphorylatable Bcl-2 mutant (Wei et al., 2008). Interestingly, JNK has also been implicated in autophagy induction in response to ER stress, oxidative stress, cancer drugs, and stimulation through the death receptor Fas; however, whether JNK mediates phosphorylation of Bcl-2 in these circumstances has not been investigated (Ogata et al., 2006; Zhang et al., 2008; Li et al., 2009; Wu et al., 2009).

In addition to this stress-induced phosphorylation of $\mathrm{Bcl}-2$ by JNK, dissociation of the Bcl-2/Bcl-xL-Beclin-1 complex and subsequent induction of autophagy can be achieved by DAPK-mediated phosphorylation of Beclin-1 on its $\mathrm{BH} 3$ domain (Zalckvar et al., 2009a, 2009b). Cells expressing a mutant Beclin-1 that mimicked phosphorylation of Thr 119 within the $\mathrm{BH} 3$ domain displayed decreased Bcl-xL-Beclin-1 binding and enhanced autophagosome formation, whereas a non-phosphorylatable Beclin-1 mutant displayed enhanced Bcl-xL binding (Zalckvar et al., 2009b). DAPK, which functions as a tumor suppressor, has been implicated in both death receptor-mediated apoptosis and autophagic cell death (Bialik and Kimchi, 2006); however, whether DAPKmediated dissociation of the Bcl-2/Bcl-xL-Beclin-1 complex serves a pro-death or pro-survival function has not yet been addressed. Thus, the enhanced autophagy observed in DAPK-overexpressing cells may instead protect cells from DAPK-mediated apoptosis.

An additional stress-related signal triggering the dissociation of the $\mathrm{Bcl}-2 / \mathrm{Bcl}-\mathrm{xL}-\mathrm{Beclin}-1$ complex involves the translocation of the damage-associated molecular pattern (DAMP) protein HMGB-1 to the cytosol. HMGB-1 translocation has long been known to occur in response to stresses including inflammation and oxidative stress; however, recent studies have demonstrated that HMGB-1 translocation also occurs in response to autophagic stimulus. Moreover, translocated HMGB-1 displaces Bcl-2 by directly interacting with Beclin-1, and decreased starvation-induced autophagy was observed upon inhibition of HMGB-1 cytosolic translocation (Kang et al., 2010; Tang et al., 2010).

Furthermore, the Bcl-2-Beclin-1 complex can be disrupted by pro-apoptotic $\mathrm{BH} 3-$ only proteins. Both Bad and Bax can disrupt the interaction between $\mathrm{Bcl}-2$ and Beclin-1; however, these proteins have opposite effects on Beclin-1-mediated autophagy (Maiuri et al., 2007; Luo and Rubinsztein, 2010). While Bad reverses the Bcl-2-mediated inhibition of autophagy, overexpression of Bax instead reduces autophagy (Maiuri et al., 2007; Luo and Rubinsztein, 2010). Although these results seem to point to opposing functions for $\mathrm{Bad}$ and Bax, the ability of Bax to inhibit autophagy may be related to 


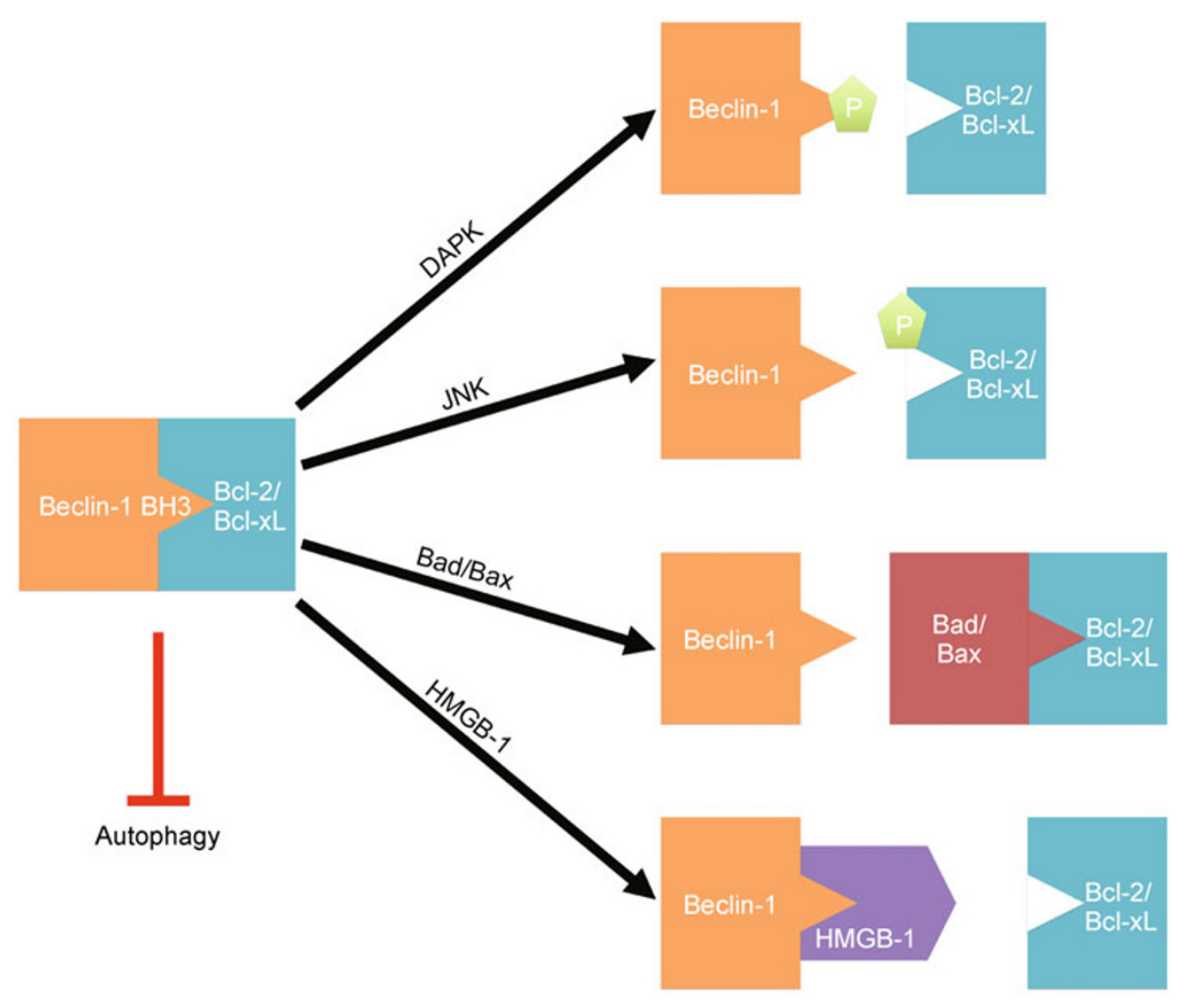

Figure 1. Release of $\mathrm{Bcl}-2 / \mathrm{Bcl}-x \mathrm{~L}-$ mediated repression of Beclin-1-induced autophagy through disruption of the $\mathrm{Bcl}-2 / \mathrm{Bcl}-$ xL-Beclin-1 interaction. Under nutrient-sufficient conditions, Beclin-1 is bound by Bcl-2 or Bcl-xL, inhibiting its ability to initiate autophagy. During starvation or other stress conditions, several mechanisms mediate the disruption of this interaction to allow autophagy. These mechanisms include DAPK-mediated phosphorylation of the BH3 domain of Beclin-1, JNK-mediated phosphorylation of the non-structured loop of Bcl-2, competition with Bad and Bax for Bcl-2/Bcl-xL binding, and binding of the DAMP molecule HMGB-1 to Beclin-1.

its ability to stimulate degradation of Beclin-1 rather than its ability to disrupt the Bcl-2-Beclin-1 complex (see next section for detailed discussion) (Luo and Rubinsztein, 2010).

Intriguingly, while the $\mathrm{Bcl}-2 / \mathrm{Bcl}-\mathrm{xL}-\mathrm{Beclin}-1$ interaction inhibits Beclin-1-dependent autophagy, it does not affect the anti-apoptotic function of Bcl-2 (Pattingre et al., 2005; Ciechomska et al., 2009b). Several possible explanations have been proposed to explain the lack of an effect of Bcl-2/ Bcl-xL-Beclin-1 binding on apoptosis, most of which involve the relatively weak affinity of $\mathrm{Bcl}-2$ for Beclin-1 in comparison to its affinity for other $\mathrm{BH} 3$-only proteins; however, further experiments are necessary to fully understand the function of the $\mathrm{Bcl}-2 / \mathrm{Bcl}-\mathrm{xL}-\mathrm{Beclin}-1$ interaction in apoptosis (Ciechomska et al., 2009a).

Although these findings overwhelmingly support a role for Beclin-1 in linking autophagy and apoptosis, the Beclin-1/ Vps34 complex is not required for autophagy in all cell types. For example, conflicting reports have raised questions regarding the role of Beclin-1 in autophagy in T lymphocytes. Beclin-1-deficient CD4+ T cells have been reported to lack autophagy and undergo increased apoptosis; however, loss of Beclin-1 in T cells does not completely abrogate LC3 punctae formation, suggesting that a Beclin-1-independent autophagy pathway exists in T cells (Arsov et al., 2011; Kovacs et al., 2011). Moreover, unlike Atg5-deficient T lymphocytes, which fail to proliferate and display increased cell death, Beclin-1-deficient $\mathrm{T}$ Iymphocytes proliferate normally (Pua et al., 2007; Arsov et al., 2011). Further supporting the existence of a Beclin-1-independent autophagy pathway, Vps34 is completely dispensable for autophagy in T lymphocytes (McLeod et al., 2011). These data demonstrate that the Beclin-1/Nps34 complex is not universally required for autophagy in T cells; thus, whether Beclin-1 serves as a bridge between apoptosis and autophagy in these cells remains unclear.

\section{APOPTOSIS TRIGGERS CLEAVAGE OF AUTOPHAGY-RELATED PROTEINS}

In addition to the ability of $\mathrm{Bcl}-2$ family proteins to regulate 
autophagy through direct interaction with Beclin-1, the effector proteases of apoptosis have also been shown to inhibit autophagy through cleavage of autophagy-related proteins. The most well known of these proteases are the cysteine-dependent aspartate-directed proteases (caspases). Caspases can be divided into effector caspases, which cleave diverse protein substrates to drive apoptosis, and initiator caspases, which cleave effector caspases, resulting in their activation, and cleave anti-apoptotic proteins, resulting in their degradation or inactivation. In addition to caspases, the calpain family of cysteine proteases, which function in diverse processes including cell motility, cell fusion, and cell cycle, also play a role in apoptotic and necrotic cell death.

Enhanced caspase 3 activity was observed in Drosophila salivary glands undergoing autophagy in 2004 (Martin and Baehrecke, 2004); however, the first direct evidence that apoptosis-associated proteases regulate the balance between apoptosis and autophagy was published in 2006, when Yousefi and colleagues detected calpain 1- and calpain 2-mediated cleavage of Atg5 in human neutrophils undergoing spontaneous apoptosis (Yousefi et al., 2006). An Nterminal Atg5 cleavage product was detected in multiple cell types and found to translocate to the mitochondria, where it associated with $\mathrm{Bcl}-\mathrm{xL}$ to induce cytochrome $\mathrm{c}$ release (Yousefi et al., 2006). Furthermore, overexpression of this $\mathrm{N}$-terminal Atg5 cleavage product was sufficient to cause nuclear condensation, demonstrating the ability of cleaved Atg5 to directly induce apoptosis (Yousefi et al., 2006). In contrast, the $\mathrm{N}$-terminal cleavage product was not sufficient to induce autophagy (Yousefi et al., 2006). Importantly, these findings explain the ability of Atg5 overexpression to sensitize tumor cells to apoptosis-inducing anti-cancer treatments such as ceramide, etoposide, doxorubicin, and CD95 (Yousefi et al., 2006). As overexpression of LC3 had no effect on apoptosis, the enhanced sensitivity to apoptosis observed in Atg5-overexpressing cells was due not to enhanced autophagy, but solely to the pro-apoptotic function of the $\mathrm{N}$-terminal Atg5 fragment generated through calpain-mediated cleavage (Yousefi et al., 2006).

Later studies further confirmed the role of autophagyrelated protein cleavage in linking apoptosis and autophagy by demonstrating that similar to calpains, caspases also cleaved autophagy proteins, including Beclin-1. Studies in Drosophila demonstrated that the caspase ortholog Dcp-1 regulates autophagy both in cultured cells and in the Drosophila ovary (Hou et al., 2008b, 2009). In mammalian cells, both initiator and effector caspases have been shown to cleave autophagy-related proteins. Similar to the calpainmediated cleavage of Atg5, Beclin-1 is cleaved by caspases following either intrinsic or extrinsic apoptotic stimulation (Cho et al., 2009; Luo and Rubinsztein, 2010; Norman et al., 2010; Wirawan et al., 2010; Zhu et al., 2010; Li et al., 2011; Rohn et al., 2011). The identity of the caspases involved in Beclin-1 cleavage may vary in different cell types or experimental systems; caspases $3,6,9$, and 10 have all been reported to be involved (Cho et al., 2009; Luo and Rubinsztein, 2010; Norman et al., 2010; Wirawan et al., 2010; Zhu et al., 2010; Li et al., 2011; Rohn et al., 2011).

Unlike Atg5, however, overexpression of full-length Beclin1 stimulates high levels of autophagy while inhibiting apoptosis, and siRNA knockdown of Beclin-1 in Hela cells resulted in increased sensitivity to tumor necrosis factorrelated apoptosis-inducing ligand (TRAIL)-induced cell death (Cho et al., 2009). The results of several recent studies suggest that the balance between cleaved and full-length Beclin-1 is reflected in the balance between apoptotic and autophagic cell fates. Only full-length Beclin-1 can induce autophagy, as in cells ectopically expressing a C-terminal Beclin-1 cleavage product, both growth factor withdrawal and staurosporine treatment instead resulted in enhanced apoptosis (Cho et al., 2009; Wirawan et al., 2010; Zhu et al., 2010). This pro-apoptotic effect appears to be directly mediated by cleaved Beclin-1, as a C-terminal cleavage product of Beclin1 translocated to the mitochondria and induced the release of cytochrome c (Wirawan et al., 2010).

While these results clearly demonstrate a role for caspasemediated Beclin-1 cleavage in the induction of apoptosis, some degree of Beclin-1 cleavage may also be required for autophagy, as Hela cells expressing non-cleavable Beclin-1 mutants failed to upregulate autophagosome formation upon starvation (Zhu et al., 2010). Furthermore, expression of noncleavable Beclin-1 failed to restore autophagosome formation or inhibit cell death in cells expressing shRNA specific for Beclin-1 (Zhu et al., 2010). In contrast, expression of noncleavable Beclin-1 did rescue the decreased autophagy observed in cells overexpressing Bax, suggesting that a fine balance between cleaved and full-length Beclin-1 is required to maintain the balance between apoptosis and autophagy (Luo and Rubinsztein, 2010). Moreover, cleavage of Beclin-1 disrupts its interaction with Bcl-2; the C-terminal Beclin-1 cleavage product maintains a weak interaction with $\mathrm{Bcl}-2$, while the interaction between $\mathrm{Bcl}-2$ and the $\mathrm{N}$-terminal cleavage product is completely lost (Zhu et al., 2010). Expression of the $\mathrm{N}$-terminal cleavage product but not the C-terminal cleavage product partially restored autophagosome formation in Beclin-1 shRNA-expressing cells, suggesting that the caspase-mediated disruption of the Beclin-1-Bcl-2 complex may serve to promote autophagy (Zhu et al., 2010).

Similarly, caspase 3-mediated cleavage of the autophagyrelated endopeptidase Atg4D was observed in Hela and A431 cells treated with staurosporine (Betin and Lane, 2009). The cleaved form of Atg4D was further shown to cleave and delipidate GABARAP-L1, resulting in decreased numbers of GABARAP-L1 punctae but not LC3 punctae (Betin and Lane, 2009). Interestingly, Atg4D was shown to localize to the mitochondria before cell death regardless of its cleavage status in a subset of staurosporine-treated cells; however, the 


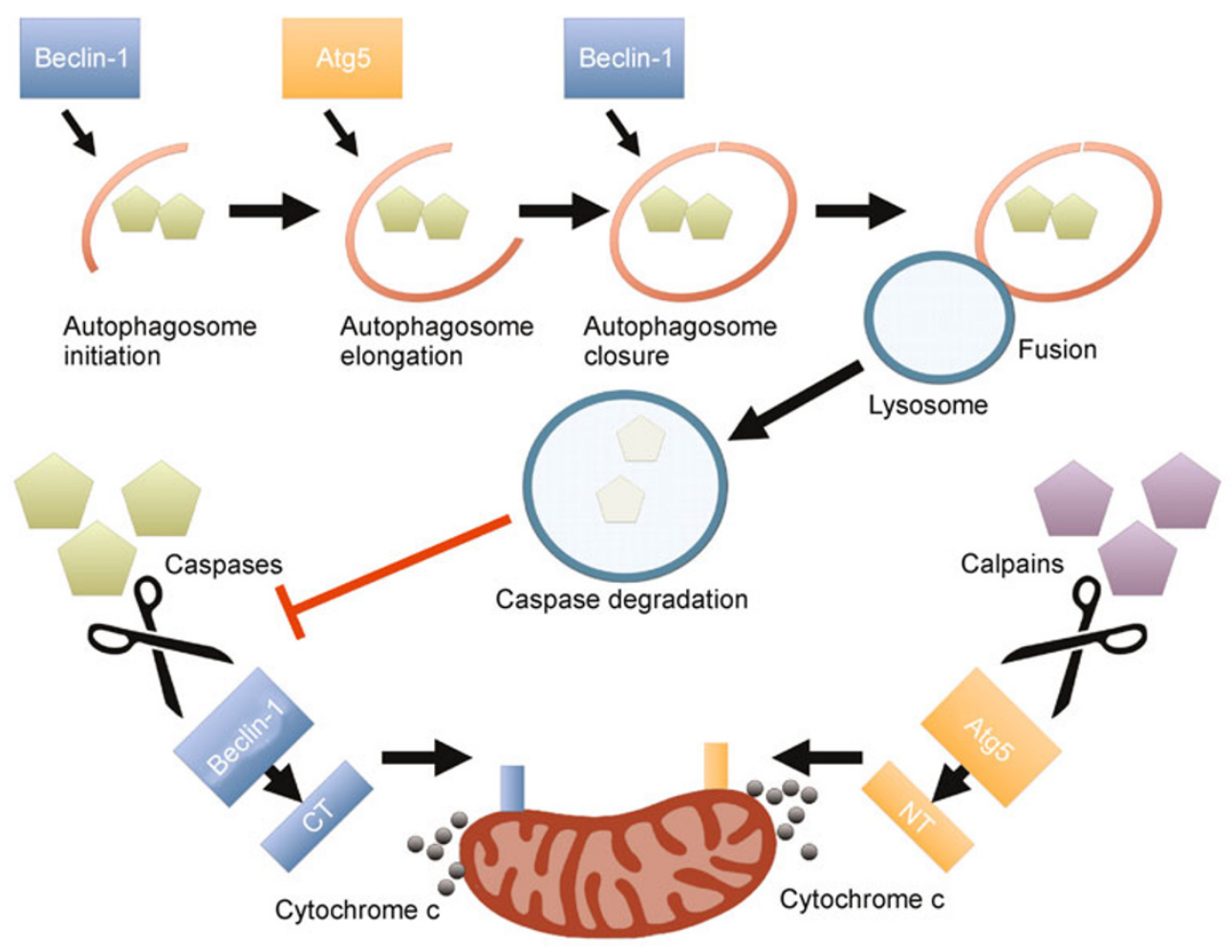

Figure 2. Mechanisms mediating the mutual negative regulation of autophagy and apoptosis. Beclin-1 is required for autophagosome initiation and autophagosome closure, while Atg5 is required for autophagosome elongation. The autophagic functions of both of these proteins are negatively regulated by apoptotic signaling. Beclin-1 is cleaved by caspases, and Atg 5 is cleaved by calpain-1 and calpain-2. These cleavage events serve not only to inhibit autophagy, but also to enhance apoptosis, as a C-terminal Beclin-1 cleavage product (CT) and an N-terminal Atg5 cleavage product (NT) are each targeted to the mitochondria, where they directly induce the release of cytochrome c. Full-length Beclin-1 and Atg5 oppose this process by mediating the autophagic degradation of active caspase 8.

implications of Atg4D mitochondrial localization remain unclear, as apoptosis occurred in staurosporine-treated cells in which Atg4D was not observed at the mitochondria (Betin and Lane, 2009).

Together, these findings suggest a common process whereby autophagy-related proteins are cleaved by apoptosis-related proteases, resulting in a shift in the balance between autophagy and apoptosis. The ability of at least some of these cleaved proteins to directly mediate apoptotic functions, such as the release of mitochondrial cytochrome $\mathrm{c}$, demonstrates the complex interactions underlying this balance. Further studies of the effects of proteolytic modification of autophagy-related proteins will not only provide additional insight into the regulation of autophagy by pro-apoptotic stimuli but also identify novel pro-apoptotic functions of modified autophagy-related proteins.

\section{CROSSTALK BETWEEN THE EXTRINSIC APOPTOTIC PATHWAY AND AUTOPHAGY}

In addition to the ability of caspases and calpains to regulate autophagy, recent studies have suggested a link between the upstream signals that induce apoptosis through the extrinsic pathway. Extrinsic apoptosis is initiated by the binding of death receptors to their cognate ligands, which results in the formation of a death-inducing signaling complex (DISC) nucleated by the adapter protein Fas-associated protein with death domain (FADD) and including the initiator caspase pro-caspase 8 . Recruitment to the DISC results in cleavage of pro-caspase 8 and release of active caspase 8 , which subsequently cleaves and activates downstream targets, including effector caspases. The DISC may also contain one or both isoforms of cellular FLICE-like inhibitory protein (cFLIP), which depending upon the context, can serve to either inhibit or enhance caspase 8 activation (Thome and Tschopp, 2001; Wilson et al., 2009).

In addition to these well-defined roles in extrinsic apoptosis, studies of cells with inactivated caspase 8 or defective FADD-pro-caspase 8 interactions have suggested that the key components of the DISC also modulate autophagy. Inhibition of or deficiency in caspase 8 results in excessive autophagy in fibroblasts, macrophages, and T cells (Yu et al., 
2004; Bell et al., 2008). Similarly, expression of a FADD mutant (FADD-DD) that contains the death domain (DD), which allows it to be recruited to the DISC, but not the death effector domain (DED), which mediates its interaction with pro-caspase 8 and c-FLIP, inhibits death receptor-induced apoptosis and induces excess levels of autophagy in epithelial cells and T cells (Thorburn et al., 2005; Bell et al., 2008).

Moreover, Atg5 has been shown to interact with FADD through its DD; however, FADD deficiency does not affect autophagosome formation (Pyo et al., 2005). Instead, the loss of FADD rescued the increased caspase-dependent cell death observed upon overexpression of Atg5, suggesting that the Atg5-FADD interaction may serve to regulate apoptosis rather than autophagy (Pyo et al., 2005).

Further evidence for crosstalk between the extrinsic apoptotic pathway and autophagy was provided by the identification of Kaposi's sarcoma-associated herpesvirus (KSHV)-associated viral FLICE-like inhibitory protein (v-FLIP) in a screen for viral proteins that inhibit autophagy (Lee et al., 2009). Similar to c-FLIP, v-FLIP inhibits death receptormediated apoptosis by preventing pro-caspase 8 cleavage.

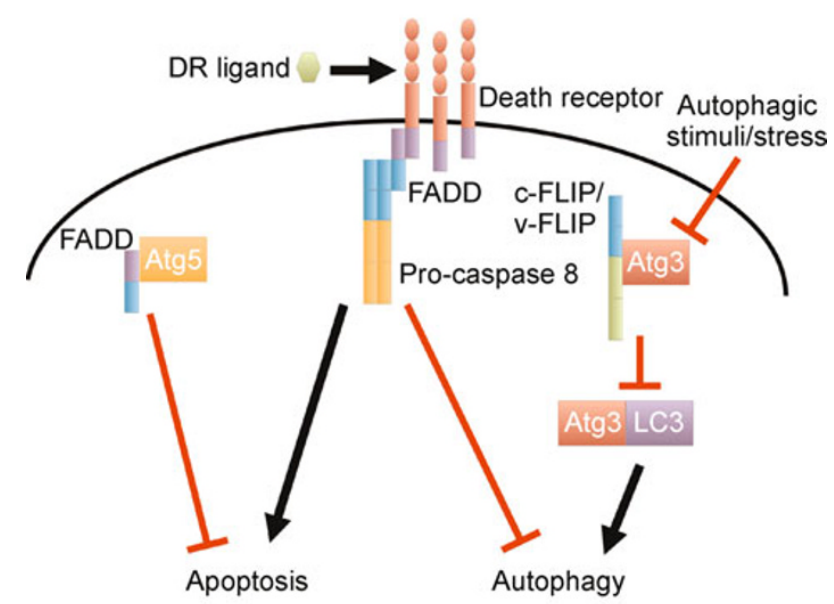

Figure 3. Crosstalk between the extrinsic apoptotic and autophagic pathways. Binding of a death receptor to its ligand results in the recruitment of pro-caspase 8 to the DISC through its interaction with the adapter protein FADD. Pro-caspase 8 cleavage results in the relase of active caspase 8 , which mediates apoptosis and inhibits autophagy through an unknown mechanism. The caspase 8 inhibitory proteins c-FLIP and vFLIP interact with Atg3, thereby preventing interaction between Atg3 and LC3 and inhibiting autophagy. The FLIP/Atg3 interaction is inhibited by starvation, allowing autophagy to proceed. Similarly, FADD directly interacts with Atg5; however, this interaction appears to inhibit apoptosis rather than autophagy.
Overexpression of KSHV v-FLIP, HVS v-FLIP, MCV 159L v-FLIP, c-FLIP ${ }_{S}$, and C-FLIP $\mathrm{L}_{\mathrm{L}}$ inhibited starvation- and rapamycin-induced autophagy, and siRNA-mediated knockdown of c-FLIP resulted in enhanced autophagy in response to rapamycin (Lee et al., 2009). This anti-autophagic function was independent of the anti-apoptotic and cellular activation functions of FLIP, as mutant v-FLIP constructs lacking the ability to bind FADD or TRAF2 or to activate NF-KB retained the ability to inhibit autophagy (Lee et al., 2009). Both c-FLIP and v-FLIP were shown to interact with Atg3, in turn preventing its interaction with LC3 and the induction of autophagy (Lee et al., 2009). Moreover, mutations in v-FLIP that prevented its interaction with Atg3 abolished its ability to inhibit autophagy (Lee et al., 2009). Interestingly, this interaction was inhibited by pro-autophagic stimuli such as starvation and rapamycin treatment, suggesting that a fine balance between c-FLIP and Atg3 expression levels may mediate cell fate decisions (Lee et al., 2009).

In each of these studies, the excess autophagy observed in cells with inhibited extrinsic apoptotic pathways was associated with increased cell death (Yu et al., 2004; Pyo et al., 2005; Bell et al., 2008; Lee et al., 2009); however, there is little evidence to suggest that autophagy is the direct cause of death of these cells. Although data demonstrating that Atg5 is required for both autophagosome formation and cell death in response to IFN-y stimulation are convincing, the finding that an $\mathrm{N}$-terminal calpain cleavage product of Atg5 can directly induce apoptosis raises an alternate explanation in which Atg5-mediated promotion of cell death may be independent of its autophagic function (Pyo et al., 2005; Yousefi et al., 2006).

Moreover, while the components of the DISC cooperate to regulate apoptosis, these findings suggest that these proteins may have separate roles in the regulation of autophagy. The enhanced autophagy observed in cells expressing FADD-DD appears to be due solely to the inability of FADD-DD to bind pro-caspase 8 , as V-FLIP inhibited autophagy regardless of its ability to bind FADD (Thorburn et al., 2005; Bell et al., 2008; Lee et al., 2009). Thus, it appears that pro-caspase 8 must be recruited to the DISC to inhibit autophagy; considering the role of downstream caspases in the cleavage of autophagyrelated proteins, caspase 8 activation may also be required. In contrast, C-FLIP (and V-FLIP) appear to play a DISCindependent role in the inhibition of autophagy; however, additional imaging studies will be necessary to determine the localization of c-FLIP during its interaction with Atg3.

\section{AUTOPHAGIC DEGRADATION OF APOPTOTIC PROTEINS: IMPLICATIONS FOR CANCER TREATMENT}

While many studies have focused on the modulation of autophagy by apoptotic signaling pathways, it is also clear that autophagy modulates apoptosis. In particular, several studies have demonstrated that autophagy inhibits apoptosis 
through the degradation of pro-apoptotic proteins including caspases. That autophagy and apoptosis play opposing roles in cancer cells was suggested by the findings that inhibition of autophagy by $3 \mathrm{MA}$ and/or chloroquine sensitized apoptosisresistant tumor cells to cancer treatment in chronic myelogenous leukemia (CML) cell lines, primary CML, and a mouse model of lymphoma (Amaravadi et al., 2007; Carew et al., 2007).

Recent studies have addressed the role of autophagy in the resistance of tumor cells to the pro-apoptotic effects of TRAIL (Han et al., 2008; Hou et al., 2008a, 2010). Much attention has been focused on the use of TRAIL as a cancer therapeutic, as unlike most TNF superfamily members, its pro-apoptotic effects are largely specific to tumor cells (French and Tschopp, 1999; Walczak et al., 1999); however, the efficacy of TRAIL treatment has been hampered by the development of TRAIL resistance in many tumors (Bhojani et al., 2003). Interestingly, recent findings suggest that the failure of TRAIL to kill resistant tumor cells may be due at least in part to the cytoprotective effects of autophagy, as Hct116 colon carcinoma cells overexpressing c-FLIP, which are resistant to TRAIL-mediated apoptosis, display enhanced expression of Beclin-1 and increased autophagosome formation upon stimulation through either TRAILRs or Fas (Han et al., 2008). Moreover, inhibition of autophagy in c-FLIP-overexpressing or Bax-deficient Hct116 cells or in naturally TRAIL-resistant tumor cells rendered them sensitive to TRAIL-mediated killing (Han et al., 2008; Hou et al., 2008a).

Additional evidence for the cytoprotective role of autophagy in tumor cells is provided by the finding that overexpression of Beclin-1 in Hela cells reduces TRAIL-mediated death (Cho et al., 2009). The susceptibility of untransfected Hela cells to TRAIL can be attributed to their failure to induce autophagy upon TRAIL stimulation, as caspase-mediated cleavage of Beclin-1 prevents the induction of LC3 punctae in TRAIL-stimulated cells (Cho et al., 2009).

Further study of the mechanisms by which autophagy prevents TRAIL-mediated death in tumor cells revealed that upon proteolytic activation of pro-caspase 8 in apoptosisresistant tumor cells, active caspase 8 was targeted to autophagosomes and subsequently degraded in lysosomes, preventing activation of downstream apoptotic effectors (Hou et al., 2010). Thus, apoptosis and autophagy each serve as a check on the other, as autophagy targets caspases for degradation, and caspases cleave autophagy-related proteins. Whether other apoptotic proteases that cleave autophagic proteins, such as calpains, can also be targeted for autophagic degradation remains unknown.

Importantly, these findings may explain the specificity of TRAIL-mediated apoptosis for tumor cells, as a similar cytoprotective autophagic response to TRAIL treatment was observed in untransformed breast epithelial cells (HerreroMartín et al., 2009). In these cells, TRAIL-induced autophagy is triggered by the transforming growth factor- $\beta$-activating kinase 1-dependent AMP-activated protein kinase (AMPK)mediated phosphorylation of mammalian target of rapamycin complex 1 (mTORC1) (Herrero-Martín et al., 2009). Both AMPK and TAK-1 are ubiquitously expressed and involved in cellular metabolism; however, whether they function in autophagy in TRAIL-resistant tumor cells is not known.

Importantly, the use of autophagy by tumor cells to subvert apoptosis is not limited to TRAIL, as similar cytoprotective autophagic responses were observed in lung cancer cells treated with the epidermal growth factor receptor tyrosine kinase inhibitors gefitinib and erlotinib, in colon cancer cells treated with the COX-2 inhibitor celecoxib, and in gastric cancer cells treated with the chemopreventive quercetin; however, whether this protection from apoptosis is the result of autophagic degradation of caspases remains unclear (Huang and Sinicrope, 2010; Han et al., 2011; Wang et al., 2011).

Together, these findings not only elucidate the mechanisms by which autophagy opposes apoptosis in tumor cells, but also suggest the potential utility-and potential danger-of combination cancer therapies targeting both apoptosis and autophagy. Further studies of the pathways involved in cytoprotective autophagy in TRAIL-resistant tumor cells and in normal, untransformed cells will provide insight into common and unique events that lead to autophagy in response to TRAIL, potentially allowing the development of combination therapies that target autophagic pathways in TRAIL-resistant tumors but not in normal cells.

\section{CONCLUSION}

Although a relationship between autophagy and cell death has long been suggested, the identification of the molecular players involved in both apoptosis and autophagy has resulted in an explosion of data defining the interactions between these pathways. Interestingly, in contrast to the initial descriptions of "autophagic cell death," accumulating evidence points to antagonistic roles for apoptosis and autophagy. While much remains to be learned about the extensive crosstalk between these two pathways, recent and ongoing research has provided insight into the physiologic roles of these processes. Continued progress in this field will be critical in developing new cancer therapies and improving those already in use.

\section{ABBREVIATIONS}

AMPK, AMP-activated protein kinase; DAMP, damage-associated molecular pattern protein; DAPK, death-associated protein kinase; $D D$, death domain; DED, death effector domain; DISC, deathinducing signaling complex; FADD, Fas-associated protein with death domain; HMGB1, high-mobility group box 1; KSHV, Kaposi's sarcoma-associated herpesvirus; TRAIL, tumor necrosis factorrelated apoptosis-inducing ligand; v-FLIP, viral FLICE-like inhibitory protein 


\section{REFERENCES}

Aita, V.M., Liang, X.H., Murty, V.V., Pincus, D.L., Yu, W., Cayanis, E., Kalachikov, S., Gilliam, T.C., and Levine, B. (1999). Cloning and genomic organization of beclin 1, a candidate tumor suppressor gene on chromosome 17q21. Genomics 59, 59-65.

Amaravadi, R.K., Yu, D., Lum, J.J., Bui, T., Christophorou, M.A., Evan, G.I., Thomas-Tikhonenko, A., and Thompson, C.B. (2007). Autophagy inhibition enhances therapy-induced apoptosis in a Myc-induced model of lymphoma. J Clin Invest 117, 326-336.

Anglade, P., Vyas, S., Javoy-Agid, F., Herrero, M.T., Michel, P.P., Marquez, J., Mouatt-Prigent, A., Ruberg, M., Hirsch, E.C., and Agid, Y. (1997). Apoptosis and autophagy in nigral neurons of patients with Parkinson's disease. Histol Histopathol 12, 25-31.

Arsov, I., Adebayo, A., Kucerova-Levisohn, M., Haye, J., MacNeil, M., Papavasiliou, F.N., Yue, Z., and Ortiz, B.D. (2011). A role for autophagic protein beclin 1 early in lymphocyte development. $J$ Immunol 186, 2201-2209.

Bell, B.D., Leverrier, S., Weist, B.M., Newton, R.H., Arechiga, A.F., Luhrs, K.A., Morrissette, N.S., and Walsh, C.M. (2008). FADD and caspase-8 control the outcome of autophagic signaling in proliferating T cells. Proc Natl Acad Sci U S A 105, 16677-16682.

Betin, V.M., and Lane, J.D. (2009). Caspase cleavage of Atg4D stimulates GABARAP-L1 processing and triggers mitochondrial targeting and apoptosis. J Cell Sci 122, 2554-2566.

Bhojani, M.S., Rossú, B.D., and Rehemtulla, A. (2003). TRAIL and anti-tumor responses. Cancer Biol Ther 2, S71-S78.

Bialik, S., and Kimchi, A. (2006). The death-associated protein kinases: structure, function, and beyond. Annu Rev Biochem 75, 189-210.

Carew, J.S., Nawrocki, S.T., Kahue, C.N., Zhang, H., Yang, C., Chung, L., Houghton, J.A., Huang, P., Giles, F.J., and Cleveland, J. L. (2007). Targeting autophagy augments the anticancer activity of the histone deacetylase inhibitor SAHA to overcome Bcr-Ablmediated drug resistance. Blood 110, 313-322.

Chang, N.C., Nguyen, M., Germain, M., and Shore, G.C. (2010). Antagonism of Beclin 1-dependent autophagy by BCL-2 at the endoplasmic reticulum requires NAF-1. EMBO J 29, 606-618.

Cho, D.H., Jo, Y.K., Hwang, J.J., Lee, Y.M., Roh, S.A., and Kim, J.C. (2009). Caspase-mediated cleavage of ATG6/Beclin-1 links apoptosis to autophagy in HeLa cells. Cancer Lett 274, 95-100.

Ciechomska, I.A., Goemans, C.G., and Tolkovsky, A.M. (2009a). Why doesn't Beclin 1, a BH3-only protein, suppress the anti-apoptotic function of Bcl-2? Autophagy 5, 880-881.

Ciechomska, I.A., Goemans, G.C., Skepper, J.N., and Tolkovsky, A. M. (2009b). Bcl-2 complexed with Beclin-1 maintains full antiapoptotic function. Oncogene 28, 2128-2141.

Debnath, J., Baehrecke, E.H., and Kroemer, G. (2005). Does autophagy contribute to cell death? Autophagy 1, 66-74.

Feng, W., Huang, S., Wu, H., and Zhang, M. (2007). Molecular basis of Bcl-xL's target recognition versatility revealed by the structure of $\mathrm{Bcl}-\mathrm{xL}$ in complex with the BH3 domain of Beclin-1. J Mol Biol 372, 223-235.

French, L.E., and Tschopp, J. (1999). The TRAIL to selective tumor death. Nat Med 5, 146-147.

Furuya, N., Yu, J., Byfield, M., Pattingre, S., and Levine, B. (2005). The evolutionarily conserved domain of Beclin 1 is required for Vps34 binding, autophagy and tumor suppressor function. Autophagy 1, 46-52.
Han, J., Hou, W., Goldstein, L.A., Lu, C., Stolz, D.B., Yin, X.M., and Rabinowich, H. (2008). Involvement of protective autophagy in TRAIL resistance of apoptosis-defective tumor cells. J Biol Chem 283, 19665-19677.

Han, W., Pan, H., Chen, Y., Sun, J., Wang, Y., Li, J., Ge, W., Feng, L., Lin, X., Wang, X., et al. (2011). EGFR tyrosine kinase inhibitors activate autophagy as a cytoprotective response in human lung cancer cells. PLoS One 6, e18691.

Herrero-Martín, G., Høyer-Hansen, M., García-García, C., Fumarola, C., Farkas, T., López-Rivas, A., and Jäättelä, M. (2009). TAK1 activates AMPK-dependent cytoprotective autophagy in TRAILtreated epithelial cells. EMBO J 28, 677-685.

Hou, W., Han, J., Lu, C., Goldstein, L.A., and Rabinowich, H. (2008a). Enhancement of tumor-TRAIL susceptibility by modulation of autophagy. Autophagy 4, 940-943.

Hou, W., Han, J., Lu, C., Goldstein, L.A., and Rabinowich, H. (2010). Autophagic degradation of active caspase-8: a crosstalk mechanism between autophagy and apoptosis. Autophagy 6, 891-900.

Hou, Y.C., Chittaranjan, S., Barbosa, S.G., McCall, K., and Gorski, S. M. (2008b). Effector caspase Dcp-1 and IAP protein Bruce regulate starvation-induced autophagy during Drosophila melanogaster oogenesis. J Cell Biol 182, 1127-1139.

Hou, Y.C., Hannigan, A.M., and Gorski, S.M. (2009). An executioner caspase regulates autophagy. Autophagy 5, 530-533.

Huang, S., and Sinicrope, F.A. (2010). Celecoxib-induced apoptosis is enhanced by ABT-737 and by inhibition of autophagy in human colorectal cancer cells. Autophagy 6, 256-269.

Kang, R., Livesey, K.M., Zeh, H.J., Loze, M.T., and Tang, D. (2010). HMGB1: a novel Beclin 1-binding protein active in autophagy. Autophagy 6, 1209-1211.

Kihara, A., Kabeya, Y., Ohsumi, Y., and Yoshimori, T. (2001a). Beclinphosphatidylinositol 3-kinase complex functions at the trans-Golgi network. EMBO Rep 2, 330-335.

Kihara, A., Noda, T., Ishihara, N., and Ohsumi, Y. (2001b). Two distinct Vps34 phosphatidylinositol 3-kinase complexes function in autophagy and carboxypeptidase $Y$ sorting in Saccharomyces cerevisiae. J Cell Biol 152, 519-530.

Klionsky, D.J. (2007). Autophagy: from phenomenology to molecular understanding in less than a decade. Nat Rev Mol Cell Biol 8, 931-937.

Kovacs, J.R., Li, C., Yang, Q., Li, G., Garcia, I.G., Ju, S., Roodman, D. G., Windle, J.J., Zhang, X., and Lu, B. (2011). Autophagy promotes T-cell survival through degradation of proteins of the cell death machinery. Cell death and differentiation. 2011 Jun 10. [Epub ahead of print].

Kroemer, G., and Levine, B. (2008). Autophagic cell death: the story of a misnomer. Nat Rev Mol Cell Biol 9, 1004-1010.

Lee, J.S., Li, Q., Lee, J.Y., Lee, S.H., Jeong, J.H., Lee, H.R., Chang, H., Zhou, F.C., Gao, S.J., Liang, C., et al. (2009). FLIP-mediated autophagy regulation in cell death control. Nat Cell Biol 11, 1355-1362.

Li, D.D., Wang, L.L., Deng, R., Tang, J., Shen, Y., Guo, J.F., Wang, Y., Xia, L.P., Feng, G.K., Liu, Q.Q., et al. (2009). The pivotal role of cJun NH2-terminal kinase-mediated Beclin 1 expression during anticancer agents-induced autophagy in cancer cells. Oncogene 28, 886-898.

Li, H., Wang, P., Sun, Q., Ding, W.X., Yin, X.M., Sobol, R.W., Stolz, D. B., Yu, J., and Zhang, L. (2011). Following cytochrome c release, 
autophagy is inhibited during chemotherapy-induced apoptosis by caspase 8-mediated cleavage of Beclin 1. Cancer Res 71, 3625-3634.

Liang, X.H., Jackson, S., Seaman, M., Brown, K., Kempkes, B., Hibshoosh, H., and Levine, B. (1999). Induction of autophagy and inhibition of tumorigenesis by beclin 1. Nature 402, 672-676.

Liang, X.H., Kleeman, L.K., Jiang, H.H., Gordon, G., Goldman, J.E., Berry, G., Herman, B., and Levine, B. (1998). Protection against fatal Sindbis virus encephalitis by beclin, a novel Bcl-2-interacting protein. J Virol 72, 8586-8596.

Liu, Y., Schiff, M., Czymmek, K., Tallóczy, Z., Levine, B., and DineshKumar, S.P. (2005). Autophagy regulates programmed cell death during the plant innate immune response. Cell 121, 567-577.

Luo, S., and Rubinsztein, D.C. (2007). Atg5 and Bcl-2 provide novel insights into the interplay between apoptosis and autophagy. Cell Death Differ 14, 1247-1250.

Luo, S., and Rubinsztein, D.C. (2010). Apoptosis blocks Beclin 1dependent autophagosome synthesis: an effect rescued by Bcl-xL. Cell Death Differ 17, 268-277.

Maiuri, M.C., Le Toumelin, G., Criollo, A., Rain, J.C., Gautier, F., Juin, P., Tasdemir, E., Pierron, G., Troulinaki, K., Tavernarakis, N., et al. (2007). Functional and physical interaction between $\mathrm{Bcl}-\mathrm{X}(\mathrm{L})$ and $\mathrm{a}$ BH3-like domain in Beclin-1. EMBO J 26, 2527-2539.

Martin, D.N., and Baehrecke, E.H. (2004). Caspases function in autophagic programmed cell death in Drosophila. Development 131, 275-284.

Matsuura, A., Tsukada, M., Wada, Y., and Ohsumi, Y. (1997). Apg1p, a novel protein kinase required for the autophagic process in Saccharomyces cerevisiae. Gene 192, 245-250.

McLeod, I.X., Zhou, X., Li, Q.J., Wang, F., and He, Y.W. (2011). The Class III Kinase Vps34 Promotes T Lymphocyte Survival through Regulating IL-7Ra Surface Expression. J Immunol 187, 5051-5061.

Meléndez, A., Tallóczy, Z., Seaman, M., Eskelinen, E.L., Hall, D.H., and Levine, B. (2003). Autophagy genes are essential for dauer development and life-span extension in C. elegans. Science 301, 1387-1391.

Norman, J.M., Cohen, G.M., and Bampton, E.T. (2010). The in vitro cleavage of the hAtg proteins by cell death proteases. Autophagy 6, 1042-1056.

Oberstein, A., Jeffrey, P.D., and Shi, Y. (2007). Crystal structure of the Bcl-XL-Beclin 1 peptide complex: Beclin 1 is a novel BH3-only protein. J Biol Chem 282, 13123-13132.

Ogata, M., Hino, S., Saito, A., Morikawa, K., Kondo, S., Kanemoto, S., Murakami, T., Taniguchi, M., Tanii, I., Yoshinaga, K., et al. (2006). Autophagy is activated for cell survival after endoplasmic reticulum stress. Mol Cell Biol 26, 9220-9231.

Otto, G.P., Wu, M.Y., Kazgan, N., Anderson, O.R., and Kessin, R.H. (2004). Dictyostelium macroautophagy mutants vary in the severity of their developmental defects. J Biol Chem 279, 15621-15629.

Pattingre, S., Tassa, A., Qu, X., Garuti, R., Liang, X.H., Mizushima, N., Packer, M., Schneider, M.D., and Levine, B. (2005). Bcl-2 antiapoptotic proteins inhibit Beclin 1-dependent autophagy. Cell 122, 927-939.

Pua, H.H., Dzhagalov, I., Chuck, M., Mizushima, N., and He, Y.W. (2007). A critical role for the autophagy gene Atg5 in T cell survival and proliferation. J Exp Med 204, 25-31.

Pyo, J.O., Jang, M.H., Kwon, Y.K., Lee, H.J., Jun, J.I., Woo, H.N.,
Cho, D.H., Choi, B., Lee, H., Kim, J.H., et al. (2005). Essential roles of Atg5 and FADD in autophagic cell death: dissection of autophagic cell death into vacuole formation and cell death. J Biol Chem 280, 20722-20729.

Rohn, T.T., Wirawan, E., Brown, R.J., Harris, J.R., Masliah, E., and Vandenabeele, P. (2011). Depletion of Beclin-1 due to proteolytic cleavage by caspases in the Alzheimer's disease brain. Neurobiol Dis 43, 68-78.

Tang, D., Kang, R., Livesey, K.M., Cheh, C.W., Farkas, A., Loughran, P., Hoppe, G., Bianchi, M.E., Tracey, K.J., Zeh, H.J. 3rd, et al. (2010). Endogenous HMGB1 regulates autophagy. J Cell Biol 190, 881-892.

Thome, M., and Tschopp, J. (2001). Regulation of lymphocyte proliferation and death by FLIP. Nat Rev Immunol 1, 50-58.

Thorburn, J., Moore, F., Rao, A., Barclay, W.W., Thomas, L.R., Grant, K.W., Cramer, S.D., and Thorburn, A. (2005). Selective inactivation of a Fas-associated death domain protein (FADD)-dependent apoptosis and autophagy pathway in immortal epithelial cells. Mol Biol Cell 16, 1189-1199.

Tsukada, M., and Ohsumi, Y. (1993). Isolation and characterization of autophagy-defective mutants of Saccharomyces cerevisiae. FEBS Lett 333, 169-174.

Walczak, H., Miller, R.E., Ariail, K., Gliniak, B., Griffith, T.S., Kubin, M., Chin, W., Jones, J., Woodward, A., Le, T., et al. (1999). Tumoricidal activity of tumor necrosis factor-related apoptosis-inducing ligand in vivo. Nat Med 5, 157-163.

Wang, K., Liu, R., Li, J., Mao, J., Lei, Y., Wu, J., Zeng, J., Zhang, T., Wu, H., Chen, L., et al. (2011). Quercetin induces protective autophagy in gastric cancer cells: involvement of Akt-mTOR- and hypoxia-induced factor 1a-mediated signaling. Autophagy 7 , 966-978.

Wei, Y., Pattingre, S., Sinha, S., Bassik, M., and Levine, B. (2008). JNK1-mediated phosphorylation of $\mathrm{Bcl}-2$ regulates starvationinduced autophagy. Mol Cell 30, 678-688.

Wilson, N.S., Dixit, V., and Ashkenazi, A. (2009). Death receptor signal transducers: nodes of coordination in immune signaling networks. Nat Immunol 10, 348-355.

Wirawan, E., Vande Walle, L., Kersse, K., Cornelis, S., Claerhout, S., Vanoverberghe, I., Roelandt, R., De Rycke, R., Verspurten, J., Declercq, W., et al. (2010). Caspase-mediated cleavage of Beclin1 inactivates Beclin-1-induced autophagy and enhances apoptosis by promoting the release of proapoptotic factors from mitochondria. Cell death \& disease 1, e18.

Wu, H., Wang, M.C., and Bohmann, D. (2009). JNK protects Drosophila from oxidative stress by trancriptionally activating autophagy. Mech Dev 126, 624-637.

Yang, Z., and Klionsky, D.J. (2010). Eaten alive: a history of macroautophagy. Nat Cell Biol 12, 814-822.

Youle, R.J., and Strasser, A. (2008). The BCL-2 protein family: opposing activities that mediate cell death. Nat Rev Mol Cell Biol 9, 47-59.

Yousefi, S., Perozzo, R., Schmid, I., Ziemiecki, A., Schaffner, T., Scapozza, L., Brunner, T., and Simon, H.U. (2006). Calpainmediated cleavage of Atg5 switches autophagy to apoptosis. Nat Cell Biol 8, 1124-1132.

Yu, L., Alva, A., Su, H., Dutt, P., Freundt, E., Welsh, S., Baehrecke, E. H., and Lenardo, M.J. (2004). Regulation of an ATG7-beclin 1 program of autophagic cell death by caspase-8. Science 304 , 
1500-1502.

Zalckvar, E., Berissi, H., Eisenstein, M., and Kimchi, A. (2009a). Phosphorylation of Beclin 1 by DAP-kinase promotes autophagy by weakening its interactions with $\mathrm{Bcl}-2$ and $\mathrm{Bcl}-\mathrm{XL}$. Autophagy 5 , 720-722.

Zalckvar, E., Berissi, H., Mizrachy, L., Idelchuk, Y., Koren, I., Eisenstein, M., Sabanay, H., Pinkas-Kramarski, R., and Kimchi, A. (2009b). DAP-kinase-mediated phosphorylation on the BH3 domain of beclin 1 promotes dissociation of beclin 1 from $\mathrm{Bcl}-\mathrm{XL}$ and induction of autophagy. EMBO Rep 10, 285-292.
Zhang, N., Hartig, H., Dzhagalov, I., Draper, D., and He, Y.W. (2005). The role of apoptosis in the development and function of $T$ lymphocytes. Cell Res 15, 749-769.

Zhang, Y., Wu, Y., Cheng, Y., Zhao, Z., Tashiro, S., Onodera, S., and Ikejima, T. (2008). Fas-mediated autophagy requires JNK activation in HeLa cells. Biochem Biophys Res Commun 377, 1205-1210.

Zhu, Y., Zhao, L., Liu, L., Gao, P., Tian, W., Wang, X., Jin, H., Xu, H., and Chen, Q. (2010). Beclin 1 cleavage by caspase-3 inactivates autophagy and promotes apoptosis. Protein cell 1, 468-477. 